\title{
Existence of a Short-Run Equilibrium of the Dixit-Stiglitz-Krugman Model
}

\author{
Minoru Tabata $\mathbb{D D}^{1}$ and Nobuoki Eshima $\mathbb{D D}^{2}$ \\ ${ }^{1}$ Department of Mathematical Sciences, Osaka Prefecture University, Osaka 599-8531, Japan \\ ${ }^{2}$ Center for Educational Outreach and Admissions, Kyoto University, Kyoto 606-8501, Japan \\ Correspondence should be addressed to Minoru Tabata; mnrtabata@luck.ocn.ne.jp
}

Received 6 March 2018; Accepted 10 April 2018; Published 25 June 2018

Academic Editor: Guang Zhang

Copyright (c) 2018 Minoru Tabata and Nobuoki Eshima. This is an open access article distributed under the Creative Commons Attribution License, which permits unrestricted use, distribution, and reproduction in any medium, provided the original work is properly cited.

\begin{abstract}
Each short-run equilibrium of the Dixit-Stiglitz-Krugman model is defined as a solution to the wage equation when the distributions of workers and farmers are given functions. We extend the discrete nonlinear operator contained in the wage equation as a set-valued operator. Applying the Kakutani fixed-point theorem to the set-valued operator, under the most general assumptions, we prove that the model has a short-run equilibrium.
\end{abstract}

\section{Introduction}

Spatial economics is an interdisciplinary field between economics and geography. In about 1990, Krugman commenced breakthrough research by placing particular emphasis on the clustering of economic activities and the formation of economic agglomeration in this interdisciplinary area. He successfully established a useful theoretical framework, which has attracted many social scientists from various disciplines. Since then, his research has grown into one of the major branches of spatial economics, which is now known as the New Economic Geography (NEG). In 2008, Krugman was awarded the Nobel Memorial Prize in Economic Sciences for his great contribution to spatial economics $[1,2]$.

A large number of discrete dynamic models are constructed in the NEG. Among such models, the Dixit-StiglitzKrugman model (DSK model) is one of the most important models (see, e.g., [3, Chapter 5], [4, 9.2], and [5, pp. 16-28]). In this model, economic activities (agriculture and manufacturing) are conducted in a set consisting of $N$ points, where $N$ is a natural number and each point represents a region. The population consists of farmers and workers. The DSK model is described by the wage equation, which is a discrete nonlinear equation of which the unknown function denotes the distribution of nominal wages $[3,(5.3)-(5.5)]$.
The DSK model has a strong nonlinearity. In fact, the wage equation has a double nonlinear singular structure in the sense that the equation contains a discrete nonlinear operator of which the kernel itself is expressed by another discrete nonlinear operator with a singularity. This nonlinearity causes great difficulty when attempting to solve the wage equation.

The insolvability of the DSK model has led to the introduction of several analytical methods in the NEG. For example, the Turing approach has been used to analyze the emergence of agglomeration in the DSK model. Moreover, several analytically solvable models have been developed in order to analyze economic agglomeration and bifurcation (see, e.g., [6, 7], [3, pp. 85-88], [5, 8-10]).

We note that the insolvability of the DSK model is increasingly problematic as the number $N$ increases. In fact, there are ample analytical results for $N=2$, whereas there are much fewer ones for $N=3$ (see, e.g., [11-14]). For $N=2$ or 3, it is customary to deal mainly with a specific case where the competition between uniform distribution and a complete agglomeration exists.

Hence, we should study the DSK model when the number $N$ is large. This article deals with existence of short-run equilibrium of the DSK model with no restriction on $N$, where each short-run equilibrium is defined as a solution to the wage equation when the distributions of workers and farmers are known functions. 
Mathematical studies on the existence of short-run equilibrium were conducted when $N=2,3[15,16]$. However, it is difficult to apply the methods used in those studies to the DSK model when $N$ is large. If restrictive conditions are imposed on the maximum of transport costs and the manufacturing expenditure, then it is proved that the DSK model has a shortrun equilibrium for every $N \geq 2$ [17, (2.10), Theorem 3.1] [18, Theorem 3.1] [19, Theorem 3]. However, these conditions are too restrictive to apply the results to various economic phenomena. Although it is important to prove that the DSK model has a short-run equilibrium for every $N \geq 2$, it has not been proved yet under general conditions.

In this paper, under the most general assumptions (Condition 1 and (7)-(10)), we prove that the DSK model has a short-run equilibrium for every $N \geq 2$. No condition is imposed on this paper in addition to these assumptions. The main result is Theorem 1 . Theorem 1(i) gives the existence of short-run equilibrium. Theorem 1(ii)(iii) gives accurate estimates for each short-run equilibrium.

This paper consists of six sections in addition to this introduction and Appendix. In Section 2, we state Condition 1 and introduce the DSK model. In Section 3, we state and discuss Theorem 1. In Section 4, we prove Theorem 1(ii)(iii). In Section 5, we extend the discrete nonlinear operator contained in the wage equation as a set-valued operator (Definition 6). In Section 6, applying the Kakutani fixed-point theorem to the set-valued operator, we prove Theorem 1(i). Section 7 is the conclusion section. In the Appendix, we prove Lemma 7, which shows that the set-valued operator satisfies the conditions of the Kakutani fixed-point theorem.

In this article, we do not use the methods developed in [17-19], and we make use neither of advanced theory of discrete nonlinear equations, of the NEG, nor of fixedpoint theory. We make use of only the Kakutani fixed-point theorem, which is one of the most fundamental fixed-point theorems (see, e.g., [20-22]). Hence, this article can be easily understood even without reading [17-19] carefully and even without having an advanced knowledge of discrete nonlinear equations, the NEG, and fixed-point theory.

\section{Condition and Equation}

By $D$ we denote a finite set consisting of $N$ points, where $N$ is an arbitrary integer such that

$$
N \geq 2 \text {. }
$$

Each point of $D$ represents a region where manufacturing and agriculture are conducted. By $L$ we denote the set of all realvalued functions $v=v(r)$ of $r \in D$. We regard $L$ as an $N$ dimensional Euclidean space. Hence, each $v=v(r) \in L$ can be regarded as a point of this Euclidean space. However, in order to avoid the confusion of elements of $L$ with points of $D$, we refer to $v=v(r) \in L$ as function of $r \in D$. We define the following norm instead of the usual Euclidean norm in $L$ :

$$
\|v\|:=\max _{s \in D}|v(s)| \text {. }
$$

We define the following closed subset of $L$ :

$$
L_{0+}:=\{v=v(r) \in L ; v(r) \geq 0 \forall r \in D\} .
$$

We divide $L_{0+}$ into two disjoint subsets as follows:

$$
L_{0+}=L_{+} \cup L_{0}
$$

where

$$
\begin{aligned}
& L_{+}:=\left\{v=v(r) \in L_{0+} ; v(r)>0 \forall r \in D\right\}, \\
& L_{0}:=\left\{v=v(r) \in L_{0+} ; v(r)=0 \text { for some } r \in D\right\} .
\end{aligned}
$$

The wage equation contains the elasticity of substitution $\sigma$, the manufacturing expenditure $\mu$, and the transport cost function $c=c(r, s)$, which is a known function of $(r, s) \in$ $D \times D$. We assume that

$$
\begin{aligned}
0 & <\mu<1, \\
\sigma & >1, \\
c(r, s) & \geq 0 \quad \forall r, s \in D, \\
c(r, s) & =c(s, r) \quad \forall r, s \in D .
\end{aligned}
$$

Moreover, the wage equation contains the distribution of workers $\lambda=\lambda(r)$ and the distribution of farmers $\phi=$ $\phi(r)$, which are known functions of $r \in D$. We assume the following condition in addition to (1) and (7)-(10).

Condition 1. One has

$$
\begin{aligned}
& \lambda=\lambda(r) \in S, \\
& \phi=\phi(r) \in S,
\end{aligned}
$$

where

$$
S:=\left\{v=v(r) \in L_{0+} ; \sum_{s \in D} v(s)=1\right\} .
$$

Condition 1, (1), and (7)-(10) are the most general assumptions (see, e.g., [3, pp. 45-49, 61-65] and [4]). No condition is imposed on this paper in addition to these assumptions. The wage equation is the following discrete nonlinear equation $[3,(5.5)]$ :

$$
w(r)=f(w)(r)^{1 / \sigma},
$$

where $w=w(r)$ denotes an unknown function that describes the distribution of nominal wages and $f=f(u)$ denotes the following discrete nonlinear operator:

$$
f(u)=f(u)(r):=\sum_{s \in D} Y(u(s)) G(u ; s)^{\sigma-1} e^{-(\sigma-1) c(r, s)} .
$$

Here $Y=Y(u(s))$ and $G=G(u ; s)$ are operators defined as follows:

$$
\begin{aligned}
Y(u(s)) & :=\mu \lambda(s) u(s)+(1-\mu) \phi(s), \\
G(u ; s)^{\sigma-1}: & =\frac{1}{\sum_{t \in D} \lambda(t)(1 / u(t))^{\sigma-1} e^{-(\sigma-1) c(s, t)}},
\end{aligned}
$$

which denote income and price index at point $s \in D$, respectively $[3,(5.3),(5.4)]$. The nonlinearity of the wage equation is fully discussed in $[19$, Section 2]. 


\section{Result and Discussion}

Noting that (17) contains the singular term $(1 / u(t))^{\sigma-1}$, we define that if $w=w(r) \in L_{+}$satisfies the wage equation (14) for all $r \in D$, then $w=w(r)$ is a solution. Each shortrun equilibrium is defined as a solution to the wage equation. Define

$$
S(\lambda):=\left\{v=v(r) \in L_{0+} ; \sum_{s \in D} \lambda(s) v(s)=1\right\} .
$$

Making use of (4), we divide $S(\lambda)$ into two disjoint subsets as follows:

$$
S(\lambda)=S_{+}(\lambda) \cup S_{0}(\lambda)
$$

where

$$
\begin{aligned}
& S_{+}(\lambda)=S(\lambda) \cap L_{+}, \\
& S_{0}(\lambda)=S(\lambda) \cap L_{0} .
\end{aligned}
$$

The following theorem is the main result, which will be proved in Sections 4 and 6 with the assist of Section 5.

Theorem 1. If Condition 1, (1), and (7)-(10) hold, then the following statements (i)-(iii) hold:

(i) The wage equation (14) has a solution $w=w(r) \in L_{+}$. then

(ii) If the wage equation (14) has a solution $w=w(r) \in L_{+}$,

$$
w=w(r) \in S_{+}(\lambda) .
$$
then

(iii) If the wage equation (14) has a solution $w=w(r) \in L_{+}$,

$$
\underline{w} \leq w(r) \leq \bar{w} \quad \forall r \in D,
$$

where

$$
\begin{aligned}
& \underline{w}:=\exp (-(\sigma-1) \mathbf{C}), \\
& \bar{w}:=\exp ((\sigma-1) \mathbf{C}), \\
& \mathbf{C}:=\max _{r, s \in D} c(r, s) .
\end{aligned}
$$

Let us compare this theorem with the previous research [17-19]. Indeed it is proved in [17-19] that (14) has a solution $w=w(r) \in L_{+}$. However, it is assumed in [17, (2.10), Theorem 3.1] that $\mu=1$ in addition to (1) and (8)-(12). It is assumed in [18, Theorem 3.1] that $\mathbf{C}$ is so small that the following inequality holds in addition to Condition 1, (1), and (7)-(10):

$$
\mathrm{C}<\frac{\log (1 / \mu)}{\sigma-1} .
$$

Moreover, it is assumed in [19, Theorem 3] that $\mu$ is so small that the following inequality holds in addition to Condition $1,(1)$, and (7)-(10):

$$
0<\mu<\frac{\min _{r \in D} \lambda(r)}{1+\min _{r \in D} \lambda(r)} .
$$

However, as mentioned in the previous section, no condition is imposed on Theorem 1 in addition to Condition $1,(1)$, and (7)-(10). We note that $\underline{w}$ and $\bar{w}$ are independent of $\mu \in(0,1)$. Hence, $(23)$ is more simple and more accurate than $[18,(5.1)$, (5.7)-(5.9), (6.1)]. Moreover, (22) was not proved in [17-19]. Applying (11) to the definition (18), we see that (22) implies that if the economy is in a short-run equilibrium, then the average of nominal wages is identically equal to 1 . Theorem 1 is an extension of previous research.

In [17-19], we directly apply the Brouwer fixed-point theorem to the discrete nonlinear operator (15). For this purpose, in [17-19] we need the restrictive conditions in addition to Condition 1, (1), and (7)-(10). In Section 5, we extend the discrete nonlinear operator (29), which is defined in the next section, as a set-valued operator (see Definition 6). By applying the Kakutani fixed-point theorem to the setvalued operator, we prove Theorem 1(i) in Section 6. By this new method, we need no restrictive condition in addition to Condition 1, (1), and (7)-(10).

\section{Necessary Conditions}

The purpose of this section is to prove Theorem 1(ii)(iii). Making use of (15), we define that if

$$
u \in L_{+},
$$

then

$$
F(u)=F(u)(r):=\frac{f(u)(r)}{u(r)^{\sigma-1}} \quad \forall r \in D .
$$

We refer to $F=F(u)$ as the wage operator. Noting that (17) and (29) contain $(1 / u(r))^{\sigma-1}$, we see that (29) can be defined for all $u \in L_{+}$, but that no $u \in L_{0}$ can be substituted in (29) (recall (5) and (6)).

We see easily that if $w=w(r) \in L_{+}$satisfies that

$$
w(r)=F(w)(r) \quad \forall r \in D,
$$

then $w=w(r)$ is a solution to (14) and that if $w=w(r) \in L_{+}$is a solution to (14), then it satisfies (30). Hence, we seek a fixed point of $F(u)$. Let us inspect (29) closely. In the next section, we make use of the following lemma.

Lemma 2. If Condition 1, (1), (7)-(10), and (28) hold, then

$$
\begin{aligned}
F(u) & \in L_{+}, \\
\lim _{U \in L_{+}, U \rightarrow u} F(U)(r) & =F(u)(r) \quad \forall r \in D .
\end{aligned}
$$

Proof. Applying (7)-(12) to (15)-(17), we see easily that

$$
\begin{gathered}
f(u) \in L_{+} \quad \forall u \in L_{+} \\
\lim _{U \in L_{+}, U \rightarrow u} f(U)(r)=f(u)(r) \quad \forall u \in L_{+} .
\end{gathered}
$$

Applying these results and (28) to (29), we obtain (31) and (32) easily.

The following lemma is the key lemma of this paper. 
Lemma 3. If Condition 1, (1), (7)-(10), and (28) hold, then

$$
\sum_{r \in D} \lambda(r) F(u)(r)=\mathbf{Y}(u),
$$

where

$$
\mathbf{Y}(u):=\sum_{s \in D} Y(u(s)) .
$$

Proof. Substituting (15) and (29) in the left-hand side of (35), we obtain

$$
\begin{gathered}
\sum_{r \in D} \lambda(r) F(u)(r)=\sum_{r \in D} \sum_{s \in D}\left\{\lambda(r)\left(\frac{1}{u(r)}\right)^{\sigma-1} Y(u(s))\right. \\
\left.\cdot G(u ; s)^{\sigma-1} e^{-(\sigma-1) c(r, s)}\right\} .
\end{gathered}
$$

Exchange $\sum_{r \in D}$ and $\sum_{s \in D}$ in the right-hand side, and apply (10) to the right-hand side. Recalling the definition (17), we see that the right-hand side of the equality thus obtained contains both $1 / G(u ; s)^{\sigma-1}$ and $G(u ; s)^{\sigma-1}$, which cancel each other out. Hence, the right-hand side is equal to (36). We obtain (35).

Let us discuss the lemma above. Noting that (15) contains (17), we see that (29) is a singular nonlinear operator expressed in terms of the double summation. Hence, the left-hand side of (35) is expressed in terms of the singular triple summation. However, the right-hand side of (35) has no singularity and is expressed in terms of the single summation of (16). Hence, the equality (35) can transform the singular triple summation into the single summation with no singularity.

Proof of Theorem 1(ii). Let $w \in L_{+}$be a solution to (14). Recalling that (14) implies (30), we substitute (30) in (35) with $u=w$. Hence we see that

$$
\sum_{r \in D} \lambda(r) w(r)=\mathbf{Y}(w) .
$$

Applying (12), (13), and (16) to (36) with $u=w$, we obtain

$$
\mathbf{Y}(w)=\mu \sum_{r \in D} \lambda(r) w(r)+(1-\mu) .
$$

Combining these equalities, making use of (7), and recalling the definitions (18) and (20), we obtain (22).

By making use of the following lemma, we prove Theorem 1(iii) (recall (24)).

Lemma 4. If Condition 1, (1), and (7)-(10) hold, and $u=$ $u(r) \in L_{+}$satisfies

$$
\begin{aligned}
& u \in S(\lambda), \\
& U_{1} \leq u(r) \leq U_{2} \quad \forall r \in D,
\end{aligned}
$$

where $U_{i}, i=1,2$, are constants such that $0<U_{1} \leq U_{2}$, then

$$
\underline{w} U_{1}^{\sigma-1} \leq f(u)(r) \leq \bar{w} U_{2}^{\sigma-1} \quad \forall r \in D .
$$

Proof. It follows from (8), (9), and (25) that

$$
\begin{aligned}
& \exp (-(\sigma-1) \mathbf{C}) \leq \exp (-(\sigma-1) c(r, s)) \leq 1 \\
& \forall r, s \in D .
\end{aligned}
$$

Applying (24), (41), and (43) to (17), we obtain

$$
\frac{U_{1}^{\sigma-1}}{\sum_{t \in D} \lambda(t)} \leq G(u ; s)^{\sigma-1} \leq \frac{\bar{w} U_{2}^{\sigma-1}}{\sum_{t \in D} \lambda(t)} \quad \forall s \in D .
$$

Applying (11) and (13) to this inequality, we obtain

$$
U_{1}^{\sigma-1} \leq G(u ; s)^{\sigma-1} \leq \bar{w} U_{2}^{\sigma-1} \quad \forall s \in D .
$$

Applying (7), (11), (12), and (13) to (16), we see that each term of $Y(u(s))$ is nonnegative for all $u(s)$

$$
\in L_{0+} \text {. }
$$

Hence, each term contained in the summation (36) is nonnegative. Applying this result, (24), (43), and (45) to (15), we obtain

$$
\underline{w} U_{1}^{\sigma-1} \mathbf{Y}(u) \leq f(u)(r) \leq \bar{w} U_{2}^{\sigma-1} \mathbf{Y}(u) \quad \forall r \in D .
$$

Applying (7), (12), (13), (16), and (18) to (36), we easily deduce that

$$
\mathbf{Y}(u)=1 \quad \forall u \in S(\lambda) .
$$

Applying this result and (40) to (47), we obtain (42).

Proof of Theorem 1(iii). By (22) we can substitute

$$
\begin{aligned}
U_{1} & :=\min _{r \in D} w(r), \\
U_{2} & :=\max _{r \in D} w(r), \\
u(r) & :=w(r),
\end{aligned}
$$

in (40)-(42). Substituting (14) in the inequality thus obtained, we see that

$$
\underline{w} U_{1}^{\sigma-1} \leq w(r)^{\sigma} \leq \bar{w} U_{2}^{\sigma-1} \quad \forall r \in D .
$$

Applying (49) to $w(r)$ contained in this inequality, we deduce that

$$
\begin{aligned}
\underline{w} U_{1}^{\sigma-1} & \leq U_{1}^{\sigma}, \\
U_{2}^{\sigma} & \leq \bar{w} U_{2}^{\sigma-1} .
\end{aligned}
$$

Solving these inequalities with respect to $U_{i}, i=1,2$, we obtain (23).

\section{Set-Valued Operator}

The purpose of this section is to extend the wage operator (29) as a set-valued operator from $S(\lambda)$ to $2^{S(\lambda)}$. Considering Theorem 1(ii), we find it reasonable to seek a fixed point of the wage operator $F(u)$ in (18). Let us obtain estimates for $F(u)$ in (20). 
Lemma 5. If Condition 1, (1), and (7)-(10) hold, then

$$
F(u) \in S_{+}(\lambda) \quad \forall u \in S_{+}(\lambda)
$$

Proof. Substitute (48) with $u \in S_{+}(\lambda)$ in the right-hand side of (35). Applying the definition (18) to the equality thus obtained, we see that

$$
F(u) \in S(\lambda) \quad \forall u \in S_{+}(\lambda)
$$

Combining this result and (31) and recalling the definition (20), we obtain (52).

Considering the definitions (5), (6), (20), and (21) and recalling that (17) and (29) contain $(1 / u(r))^{\sigma-1}$, we see that $F(u)$ can be defined for all $u \in S_{+}(\lambda) \subset L_{+}$, but that no $u \in S_{0}(\lambda) \subset L_{0}$ can be substituted in $F(u)$. Hence, in order to analyze such a singularity, we extend the wage operator $F(u)$ as a set-valued operator from $S(\lambda)$ to $2^{S(\lambda)}$. Noting (19), we define this set-valued operator, which is denoted by $\mathbf{F}=\mathbf{F}(u)$ : $S(\lambda) \rightarrow 2^{S(\lambda)}$, as follows.

Definition 6. (i)

$$
\mathbf{F}(u):=\{F(u)\} \quad \text { for every } u \in S_{+}(\lambda),
$$

(ii)

$$
\mathbf{F}(u):=\left\{v \in S_{0}(\lambda) ; D^{0}(v) \supseteq D^{+}(u)\right\}
$$

$$
\text { for every } u \in S_{0}(\lambda) \text {, }
$$

where

$$
\begin{aligned}
& D^{+}(v):=\{r \in D ; v(r)>0\}, \\
& D^{0}(v):=\{r \in D ; v(r)=0\} .
\end{aligned}
$$

\section{Convergence of a Sequence of Solutions}

The following lemma shows that the set-valued operator $\mathbf{F}(u)$ satisfies the conditions of the Kakutani fixed-point theorem.

Lemma 7. If Condition 1, (1), and (7)-(10) hold, and

$$
\lambda=\lambda(r) \in S \cap L_{+}
$$

then the following statements (i)-(vi) hold:

(i) $S(\lambda)$ is a nonempty, compact, and convex subset of the Euclidean space $L$. $S_{+}(\lambda)$.

(ii) $\mathbf{F}(u)$ is a singleton subset of $S_{+}(\lambda) \subset S(\lambda)$ for every $u \in$

(iii) $\mathbf{F}(u)$ is a nonempty subset of $S_{0}(\lambda) \subset S(\lambda)$ for every $u \in S_{0}(\lambda)$.

(iv) $\mathbf{F}(u)$ is a convex subset of $S(\lambda)$ for every $u \in S(\lambda)$.

(v) $\mathbf{F}=\mathbf{F}(u)$ has a closed graph.

(vi) $\mathbf{F}=\mathbf{F}(u)$ has no fixed point in $S_{0}(\lambda)$.

This lemma is proved in the Appendix.
Proof of Theorem 1(i). Assume that (58) holds. Making use of Lemma 7(i)-(v), we can apply the Kakutani fixed-point theorem to the set-valued function $\mathbf{F}=\mathbf{F}(u)$. Hence we see that $\mathbf{F}=\mathbf{F}(u)$ has a fixed point in $S(\lambda)$. Making use of (19) and Lemma $7(\mathrm{vi})$, we see that $\mathbf{F}=\mathbf{F}(u)$ has a fixed point $w=w(r) \in S_{+}(\lambda)$. Applying Definition 6(i) to this result, we see that the fixed point $w=w(r)$ is a solution to (30). Hence, $w=w(r)$ is a solution to (14). Hence, we obtain Theorem 1(i) when (58) holds.

By making use of Theorem 1(i) with (58), we prove Theorem 1(i) when

$$
\lambda=\lambda(r) \in S \cap L_{0} .
$$

Recall (16). We denote $f(u)$ by $f(u ; \lambda)$ in order to emphasize that (15) contains $\lambda=\lambda(x)$. Defining

$$
\lambda_{n}=\lambda_{n}(r):=\frac{\lambda(r)+1 / n}{1+N / n}, \quad \text { for each } n \in \mathbb{N}
$$

and making use of (11) and (13), we see easily that

$$
\begin{aligned}
\lambda_{n}=\lambda_{n}(r) \in S \cap L_{+}, \quad \forall n \in \mathbb{N}, \\
\left\|\lambda_{n}-\lambda\right\| \longrightarrow 0 \quad \text { as } n \longrightarrow+\infty .
\end{aligned}
$$

Substitute $\lambda(r)=\lambda_{n}(r)$ in the wage equation (14). Making use of (61), we can apply Theorem 1(i) with (58) to the wage equation thus obtained, that is, to

$$
w=f\left(w ; \lambda_{n}\right)^{1 / \sigma}
$$

for each $n \in \mathbb{N}$. Hence, we can define a sequence of solutions $\left\{w_{n}\right\}$ such that

$$
w_{n}=f\left(w_{n} ; \lambda_{n}\right)^{1 / \sigma} \quad \forall n \in \mathbb{N} .
$$

Applying (23) to this sequence of solutions, we see that

$$
w_{n}=w_{n}(r) \in L(\underline{w}, \bar{w}), \quad \forall n \in \mathbb{N},
$$

where

$$
L(\underline{w}, \bar{w}):=\{u=u(r) \in L ; \underline{w} \leq u(r) \leq \bar{w} \forall r \in D\} .
$$

Noting that $L(\underline{w}, \bar{w})$ is a compact subset of $L$, we see that $\left\{w_{n}\right\}$ contains a convergent subsequence. Denoting this subsequence by the same symbol $\left\{w_{n}\right\}$, we see that

$$
w_{\infty}:=\lim _{n \rightarrow \infty} w_{n} \in L(\underline{w}, \bar{w}) \subset L_{+} .
$$

Applying this result and (62) to (64), when $n \rightarrow+\infty$, and performing the same calculations as those when proving (34), we see easily that

$$
w_{\infty}=f\left(w_{\infty} ; \lambda\right)^{1 / \sigma} .
$$

Hence we obtain Theorem 1(i) when (59) holds.

\section{Conclusion}

Under the most general assumptions (Condition 1, (1), and (7)-(10)), we have proved that there exists a short-run equilibrium of the Dixit-Stiglitz-Krugman model (Theorem 1(i)). Moreover we have obtained the accurate estimates for each short-run equilibrium (Theorem 1(ii)(iii)). 


\section{Appendix}

Proof of Lemma 7(i)(ii). Recalling that $L$ is an $N$-dimensional Euclidean space and making use of (58), we see that (18) is a simplex contained in $L$. Hence we obtain (i) easily. Note that if (59) holds, then (18) is not a simplex. Applying (52) to Definition 6(i), we obtain (ii).

We make use of the following lemma in order to prove Lemma 7(iii)-(vi).

Lemma 8. If Condition 1, (1), (7)-(10), and (58) hold, then the following statements (i) and (ii) hold:

(i) If $u \in S_{0}(\lambda)$, then $D^{0}(u)$ and $D^{+}(u)$ are nonempty proper subsets of $D$.

(ii) For each nonempty proper subset $d \subset D$, there exists $v \in S_{0}(\lambda)$ such that $D^{0}(v) \supseteq d$.

Proof. Making use of (58) and recalling the definitions (6), (18), (21), (56), and (57), we obtain (i) easily. By making use of (58), we can define the following function $v=v(r)$ for each nonempty proper subset $d \subset D$ :

$$
\begin{aligned}
& v(r):=\frac{1}{\sum_{s \in D \backslash d} \lambda(s)} \quad \forall r \in D \backslash d, \\
& v(r):=0 \quad \forall r \in d,
\end{aligned}
$$

where $D \backslash d$ denotes the set of elements in $D$ but not in $d$. We see easily that $v \in S_{0}(\lambda)$ and $D^{0}(v)=d$.

Proof of Lemma 7(iii)(iv). Making use of Lemma 8(i), we can substitute $d=D^{+}(u)$ in Lemma 8 (ii) for each $u \in S_{0}(\lambda)$. Hence, there exists $v \in S_{0}(\lambda)$ such that

$$
D^{0}(v) \supseteq D^{+}(u) .
$$

Applying Definition 6(ii) to this result, we obtain (iii).

It follows immediately from the inclusion relation mentioned in Definition 6(ii) that $\mathbf{F}(u)$ is a convex subset of $S(\lambda)$ for every $u \in S_{0}(\lambda)$. It follows from Lemma 7 (ii) that $\mathbf{F}(u)$ is a convex subset of $S(\lambda)$ for every $u \in S_{+}(\lambda)$. Combining these results, we obtain (iv).

Proof of Lemma $7(v)$. Consider arbitrary convergent sequences,

$$
\begin{aligned}
&\left\{u^{n}\right.\left.=u^{n}(r)\right\} \subset S(\lambda), \\
&\left\{F^{n}=F^{n}(r)\right\} \subset S(\lambda),
\end{aligned}
$$

such that

$$
F^{n} \in \mathbf{F}\left(u^{n}\right) \quad \forall n \in \mathbb{N} \text {. }
$$

Making use of Lemma 7(i), we see that

$$
u^{\infty}, F^{\infty} \in S(\lambda)
$$

where

$$
\begin{aligned}
& u^{\infty}=u^{\infty}(r):=\lim _{n \rightarrow+\infty} u^{n}(r), \\
& F^{\infty}=F^{\infty}(r):=\lim _{n \rightarrow+\infty} F^{n}(r) .
\end{aligned}
$$

We have only to prove that

$$
F^{\infty} \in \mathbf{F}\left(u^{\infty}\right)
$$

Recall (19). Let us prove (A.9) when

$$
u^{\infty} \in S_{+}(\lambda) \text {. }
$$

Recalling the definitions (5) and (20), we see easily that $S_{+}(\lambda)$ is the relative interior of the simplex $S(\lambda)$. Hence, we see that there exists a positive integer $n_{0}$ such that

$$
u^{n} \in S_{+}(\lambda) \quad \forall n \geq n_{0} .
$$

Applying this result and Definition 6(i) to (A.5), we see that

$$
F^{n}=F\left(u^{n}\right) \quad \forall n \geq n_{0} .
$$

Applying these results, (A.7), (A.8), and (A.10) to (32) with $(U, u)=\left(u^{n}, u^{\infty}\right)$, we obtain

$$
F^{\infty}=F\left(u^{\infty}\right)
$$

Applying (A.10) and Definition 6(i) to this equality, we obtain (A.9).

Let us prove (A.9) when

$$
u^{\infty} \in S_{0}(\lambda)
$$

Making use of (19), we can divide (A.3) into two disjoint subsequences as follows:

$$
\left\{u^{n}\right\}=\left\{u_{+}^{n}\right\} \cup\left\{u_{0}^{n}\right\}
$$

where

$$
\begin{aligned}
& \left\{u_{+}^{n}\right\}:=\left\{u^{n}\right\} \cap S_{+}(\lambda), \\
& \left\{u_{0}^{n}\right\}:=\left\{u^{n}\right\} \cap S_{0}(\lambda) .
\end{aligned}
$$

Applying Lemma 7(ii)(iii) and Definition 6 to (A.16) and (A.17), we can divide (A.4) into two disjoint subsequences as follows:

$$
\left\{F^{n}\right\}=\left\{F_{+}^{n}\right\} \cup\left\{F_{0}^{n}\right\}
$$

where

$$
\begin{aligned}
& F_{+}^{n}:=F\left(u_{+}^{n}\right) \in S_{+}(\lambda), \quad \forall n \in \mathbb{N}, \\
& F_{0}^{n} \in \mathbf{F}\left(u_{0}^{n}\right) \subset S_{0}(\lambda), \quad \forall n \in \mathbb{N} .
\end{aligned}
$$

At least one of (A.16) and (A.17) is an infinite convergent sequence. Assume that (A.16) is an infinite convergent sequence. Applying Lemma 8(i) with $u=u^{\infty}$ to (A.14), we deduce that

$$
\begin{aligned}
& d^{\infty} \\
& \quad:=D^{0}\left(u^{\infty}\right) \text { is a nonempty proper subset of } D .
\end{aligned}
$$

Let us obtain estimates for $F\left(u_{+}^{n}\right)(r)$. Applying (2), (36), (43), and (46) to (15) with $u=u_{+}^{n}$, we see that

$$
0 \leq f\left(u_{+}^{n}\right)(r) \leq\left\|G\left(u_{+}^{n} ;\right)^{\sigma-1}\right\| \mathbf{Y}\left(u_{+}^{n}\right) \quad \forall r \in D .
$$


Applying this inequality to (29) with $u=u_{+}^{n}$, we obtain

$$
0 \leq F\left(u_{+}^{n}\right)(r) \leq \frac{\left\|G\left(u_{+}^{n} ; \cdot\right)^{\sigma-1}\right\|}{u_{+}^{n}(r)^{\sigma-1}} \mathbf{Y}\left(u_{+}^{n}\right) \quad \forall r \in D .
$$

Let us obtain estimates for $\left\|G\left(u_{+}^{n} ; \cdot\right)^{\sigma-1}\right\|$. Recall (43) and (A.21). Replacing $D$ and $\exp (-(\sigma-1) c(r, s))$ with $d^{\infty} \subset D$ and $\exp (-(\sigma-1) \mathrm{C})$, respectively, in (17) with $u=u_{+}^{n}$, we see that

$$
0 \leq G\left(u_{+}^{n} ; s\right)^{\sigma-1} \leq \frac{\exp ((\sigma-1) \mathbf{C})}{\sum_{t \in d^{\infty}} \lambda(t)\left(1 / u_{+}^{n}(t)\right)^{\sigma-1}}
$$

$\forall s \in D$.

Replacing $u_{+}^{n}(t)$ with

$$
\mathbf{U}_{n}:=\max _{t \in d^{\infty}} u_{+}^{n}(t),
$$

in the right-hand side of this inequality, we deduce that

$$
0 \leq G\left(u_{+}^{n} ; s\right)^{\sigma-1} \leq \frac{\mathbf{U}_{n}^{\sigma-1} \exp ((\sigma-1) \mathbf{C})}{\sum_{t \in d^{\infty}} \lambda(t)} \quad \forall s \in D .
$$

Noting that $\left\{u_{+}^{n}\right\}$ is an infinite convergent subsequence of $\left\{u^{n}\right\}$ and applying (57), (A.7), and (A.21) to (A.25), we see easily that

$$
\mathbf{U}_{n} \longrightarrow 0 \quad \text { as } n \longrightarrow+\infty \text {. }
$$

It follows from (58) and (A.21) that the denominator of the right-hand side of (A.26) is a positive constant independent of $n \in \mathbb{N}$. Applying this result and (A.27) to (A.26), we see that

$$
\left\|G\left(u_{+}^{n} ; \cdot\right)^{\sigma-1}\right\| \longrightarrow 0 \text { as } n \longrightarrow+\infty .
$$

Making use of (48), (A.3), and (A.16), we deduce that

$$
\mathbf{Y}\left(u_{+}^{n}\right)=1 \quad \forall n \in \mathbb{N} .
$$

Applying Lemma 8(i) to (A.14) in the same way as (A.21), we see that

$$
D^{+}\left(u^{\infty}\right) \text { is a nonempty proper subset of } D \text {. }
$$

It follows from this result, (20), (56), (A.7), and (A.16) that

$$
\inf _{n \in \mathbb{N}} u_{+}^{n}(r)>0 \quad \forall r \in D^{+}\left(u^{\infty}\right) .
$$

Substitute (A.29) in (A.23), and let $n \rightarrow+\infty$. Noting that the denominator of the right-hand side of (A.23) is equal to $u_{+}^{n}(r)^{\sigma-1}$ and noting (A.30) and (A.31), we restrict the variable $r \in D$ within $D^{+}\left(u^{\infty}\right) \subset D$. Making use of (A.7), (A.8), (A.28), and (A.31), we see that

$$
F^{\infty}(r)=0 \quad \forall r \in D^{+}\left(u^{\infty}\right)
$$

that is,

$$
D^{0}\left(F^{\infty}\right) \supseteq D^{+}\left(u^{\infty}\right)
$$

Recalling (21), (57), and (A.6) and applying (A.30) to (A.33), we see that

$$
F^{\infty} \in S_{0}(\lambda) .
$$

Applying this result, (A.14), and (A.33) to Definition 6(ii), we obtain (A.9).

Assume that (A.17) is an infinite convergent sequence. Applying Definition 6(ii) to (A.17) and (A.20), we see that

$$
D^{0}\left(F_{0}^{n}\right) \supseteq D^{+}\left(u_{0}^{n}\right) \quad \forall n \in \mathbb{N} .
$$

Note that $\left\{u_{0}^{n}\right\}$ and $\left\{F_{0}^{n}\right\}$ are infinite subsequences of $\left\{u^{n}\right\}$ and $\left\{F^{n}\right\}$, respectively. Recall the definition (56). It follows from (A.7) and (A.8) that there exists a positive integer $n_{1}$ such that if $n \geq n_{1}$, then

$$
\begin{aligned}
& D^{+}\left(u_{0}^{n}\right) \supseteq D^{+}\left(u^{\infty}\right), \\
& D^{+}\left(F_{0}^{n}\right) \supseteq D^{+}\left(F^{\infty}\right) .
\end{aligned}
$$

Taking the complements of both sides of (A.37) and recalling the definitions (56) and (57), we obtain

$$
D^{0}\left(F_{0}^{n}\right) \subseteq D^{0}\left(F^{\infty}\right) \quad \forall n \geq n_{1} .
$$

Applying (A.36) and (A.38) to (A.35), we obtain (A.33). In the same way as above we obtain (A.9). Therefore we obtain (v).

Proof of Lemma 7(vi). Assume that $\mathbf{F}=\mathbf{F}(u)$ has a fixed point in $S_{0}(\lambda)$, that is, that there exists $u \in S_{0}(\lambda)$ such that $u \in \mathbf{F}(u)$. Applying Definition 6(ii) to this assumption, we see that

$$
D^{0}(u) \supseteq D^{+}(u) .
$$

Applying Lemma 8 (i) to $u \in S_{0}(\lambda)$, we see that both sides of this inclusion relation are nonempty proper subsets of $D$. However, it follows from the definitions (56) and (57) that both sides are disjoint from each other. This is a contradiction. Hence we obtain (vi).

\section{Data Availability}

Data and materials sharing is not applicable to this article as no datasets or materials were generated or analyzed during the current study.

\section{Conflicts of Interest}

The authors declare that there are no conflicts of interest regarding the publication of this paper.

\section{Authors' Contributions}

All authors contributed equally to this article and read and approved the final manuscript.

\section{Acknowledgments}

Minoru Tabata and Nobuoki Eshima are supported in part by Grant-in-Aid for Scientific Research of Japan (15K05005, 26330045). 


\section{References}

[1] P. Krugman, "The official homepage of the Nobel Prize in Economic Sciences," 2008, http://www.nobelprize.org/nobel_ prizes/economic-sciences/laureates/.

[2] G. I. P. Ottaviano and D. Puga, "Agglomeration in the global economy: a survey of the 'new economic geography"' World Economy, vol. 21, no. 6, pp. 707-731, 1998.

[3] M. Fujita, P. R. Krugman, and A. Venables, The Spatial Economy: Cities, Regions, and International Trade, MIT Press, 2001.

[4] M. Fujita and J.-F. Thisse, Economics of Agglomeration: Cities, Industrial Location, and Globalization, Cambridge University Press, Cambridge, UK, 2013.

[5] K. Ikeda and K. Murota, Bifurcation Theory for Hexagonal Agglomeration in Economic Geography, Springer, Tokyo, 2014.

[6] T. Akamatsu, Y. Takayama, and K. Ikeda, "Spatial discounting, Fourier, and racetrack economy: a recipe for the analysis of spatial agglomeration models," Journal of Economic Dynamics and Control, vol. 36, no. 11, pp. 1729-1759, 2012.

[7] R. Forslid and G. I. P. Ottaviano, "An analytically solvable coreperiphery model," Journal of Economic Geography, vol. 3, no. 3, pp. 229-240, 2003.

[8] K. Ikeda, K. Murota, T. Akamatsu, and Y. Takayama, "Agglomeration patterns in a long narrow economy of a new economic geography model: analogy to a racetrack economy," International Journal of Economic Theory, vol. 13, no. 1, pp. 113-145, 2017.

[9] P. Krugman, The Self-Organizing Economy Blackwell Publishers, Cambridge MA, 1996.

[10] M. Pflüger, "A simple, analytically solvable, Chamberlinian agglomeration model," Regional Science \& Urban Economics, vol. 34 , no. 5, pp. 565-573, 2004.

[11] T. Ago, I. Isono, and T. Tabuchi, "Locational disadvantage of the hub," Annals of Regional Science, vol. 40, no. 4, pp. 819-848, 2006.

[12] S. B. S. D. Castro, J. Correia-da-Silva, and P. Mossay, "The core-periphery model with three regions and more," Papers in Regional Science, vol. 91, no. 2, pp. 401-418, 2012.

[13] P. Krugman and R. L. Elizondo, "Trade policy and the third world metropolis," Journal of Development Economics, vol. 49, no. 1, pp. 137-150, 1996.

[14] T. Mori and K. Nishikimi, "Economies of transport density and industrial agglomeration," Regional Science \& Urban Economics, vol. 32, no. 2, pp. 167-200, 2002.

[15] P. Mossay, "The core-periphery model: a note on the existence and uniqueness of short-run equilibrium," Journal of Urban Economics, vol. 59, no. 3, pp. 389-393, 2006.

[16] N. G. Pavlidis, M. N. Vrahatis, and P. Mossay, "Existence and computation of short-run equilibria in economic geography," Applied Mathematics and Computation, vol. 184, no. 1, pp. 93$103,2007$.

[17] M. Tabata, N. Eshima, and Y. Sakai, "Existence, uniqueness, and computation of short-run and long-run equilibria of the Dixit-Stiglitz-Krugman model in an urban setting," Applied Mathematics and Computation, vol. 234, pp. 339-355, 2014.

[18] M. Tabata, N. Eshima, Y. Kiyonari, and I. Takagi, "The existence and uniqueness of short-run equilibrium of the Dixit-Stiglitz-Krugman model in an urban-rural setting," IMA Journal of Applied Mathematics, vol. 80, no. 5, pp. 474-493, 2015.

[19] M. Tabata and N. Eshima, "Existence and uniqueness of solutions to the wage equation of Dixit-Stiglitz-Krugman Model with no restriction on transport costs," Discrete Dynamics in Nature and Society, vol. 2017, Article ID 9341502, pp. 1-7, 2017.
[20] K. C. Border, Fixed Point Theorems with Applications to Economics and Game Theory, Cambridge University Press, Cambridge, Uk, 1985.

[21] I. Farmakis and M. Moskowitz, Fixed Point Theorems and Their Applications, World Scientific, Singapore, Singapore, 2013.

[22] A. Granas and J. Dugundji, Fixed Point Theory, Springer Science \& Business Media, 2013. 


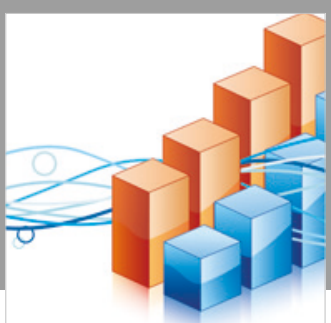

Advances in

Operations Research

\section{-n-m}
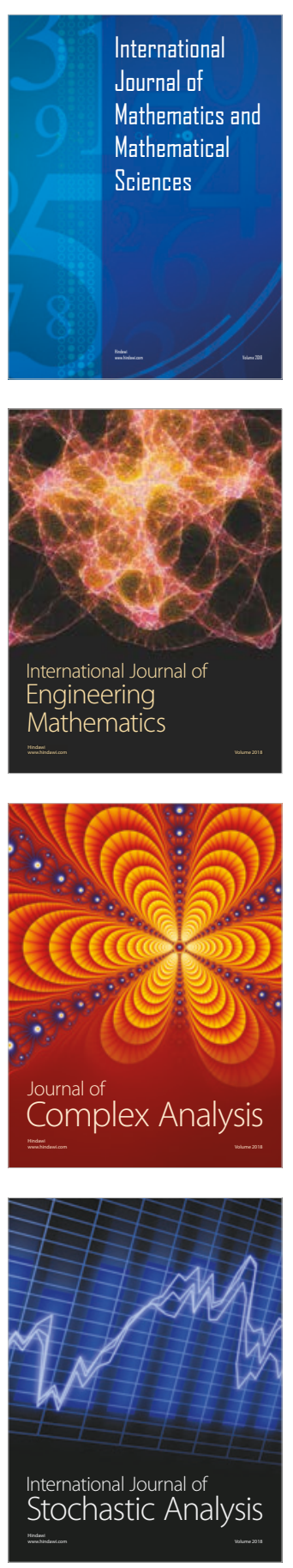
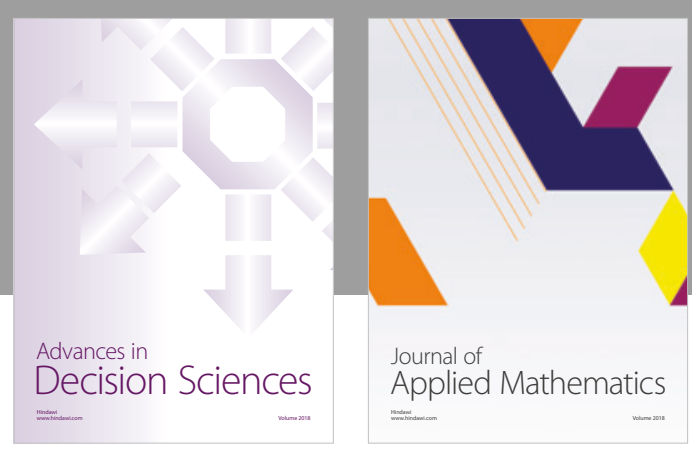

Journal of

Applied Mathematics
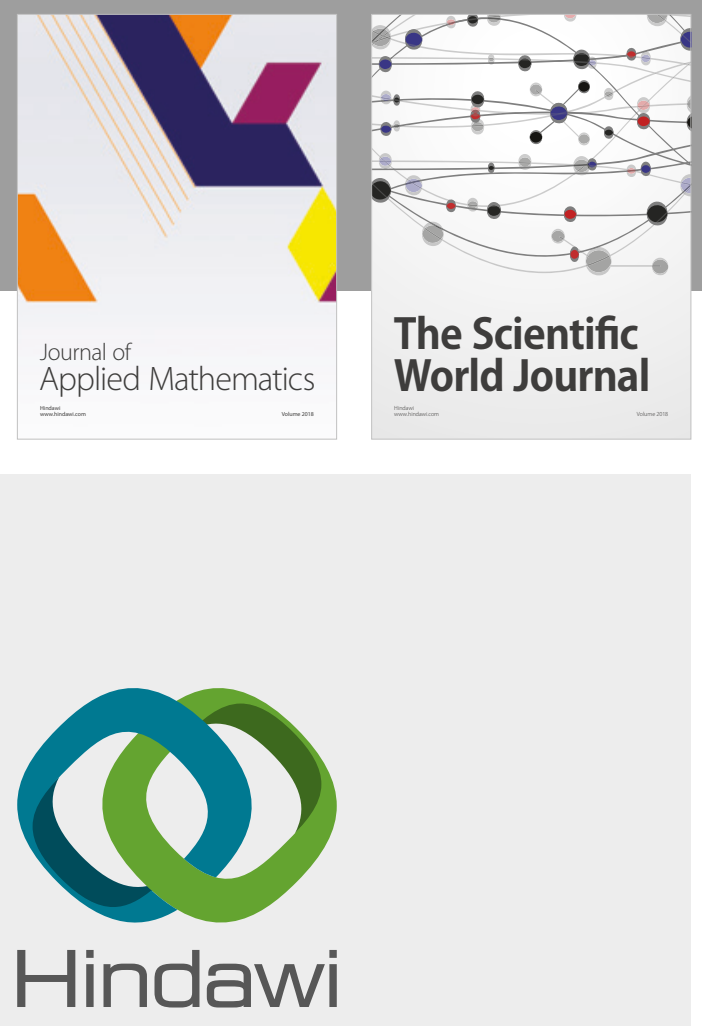

Submit your manuscripts at

www.hindawi.com

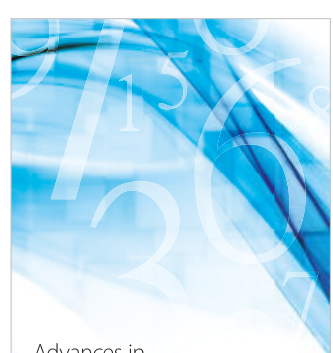

Advances in
Numerical Analysis
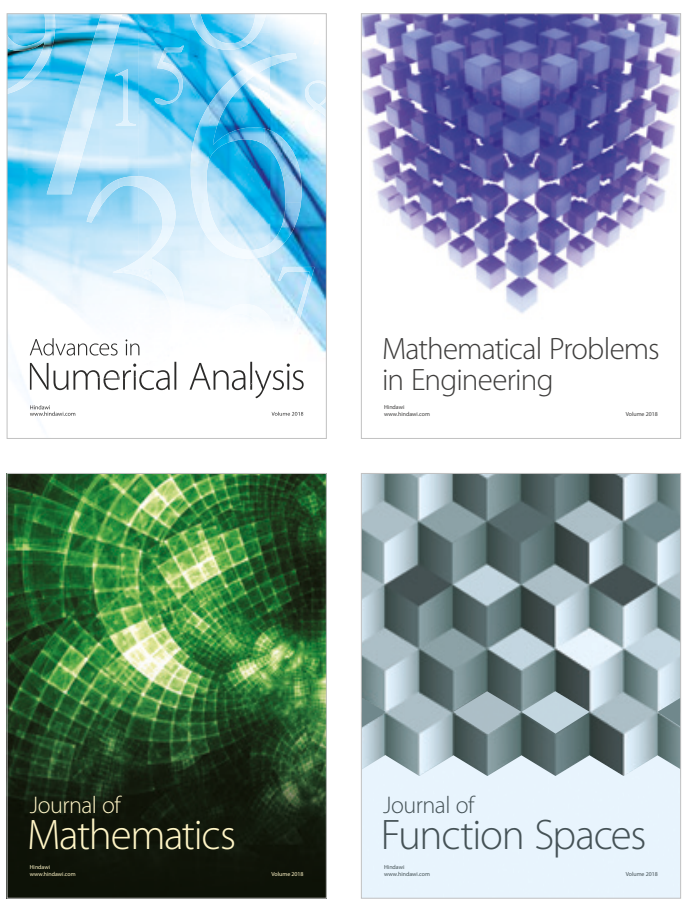

Mathematical Problems in Engineering

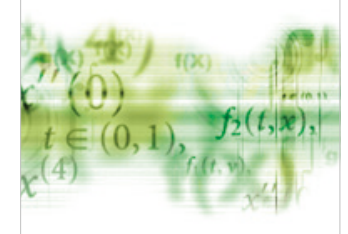

International Journal of

Differential Equations

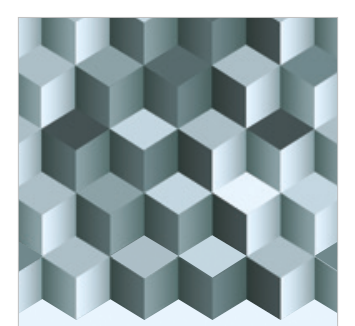

Journal of

Function Spaces

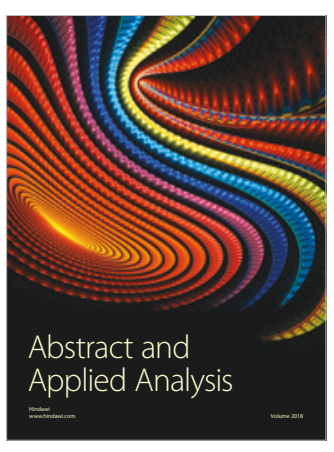

The Scientific

World Journal

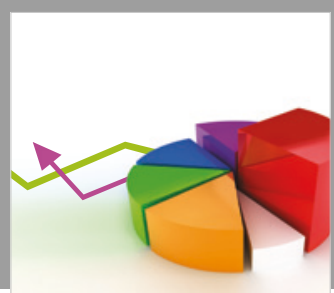

Journal of

Probability and Statistics
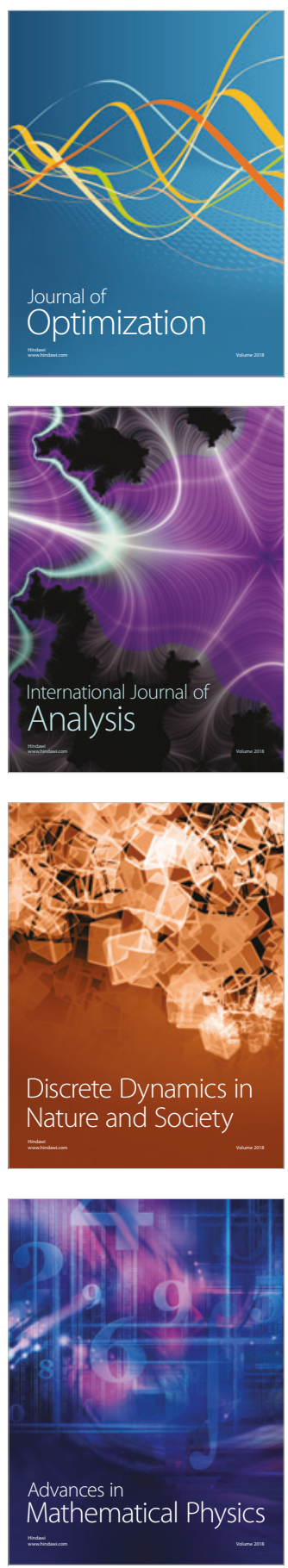